\title{
A Class of Degenerate Nonlinear Elliptic Equations in Weighted Sobolev Space
}

\author{
Rasmita Kar \\ TIFR Center for Applicable Mathematics, Karnataka, Bangalore 560065, India \\ Correspondence should be addressed to Rasmita Kar; rasmitak6@gmail.com \\ Received 9 December 2013; Accepted 12 February 2014; Published 26 March 2014 \\ Academic Editors: F. Colombini, E. Marusic-Paloka, and C. Mascia
}

Copyright (C) 2014 Rasmita Kar. This is an open access article distributed under the Creative Commons Attribution License, which permits unrestricted use, distribution, and reproduction in any medium, provided the original work is properly cited.

We prove the existence of a weak solution for the degenerate nonlinear elliptic Dirichlet boundary-value problem $L u-\mu u g_{1}+$ $h(u, \nabla u) g_{2}=f$ in $\Omega, u=0$ on $\partial \Omega$, in a suitable weighted Sobolev space, where $\Omega \subset \mathbb{R}^{n}$ is a bounded domain and $h$ is a continuous bounded nonlinearity.

\section{Introduction}

Let $\Omega \subset \mathbb{R}^{n}$ be a bounded domain with boundary $\partial \Omega$. Let $L$ be an operator in divergence form:

$$
L u(x)=-\sum_{i, j=1}^{n} D_{j}\left(a_{i j}(x) D_{i} u(x)\right) \quad \text { with } D_{j}=\frac{\partial}{\partial x_{j}},
$$

with coefficients $a_{i j} / \omega \in L^{\infty}(\Omega)$ which are symmetric and satisfy the degenerate ellipticity condition:

$$
\lambda|\xi|^{2} \omega(x) \leq \sum_{i, j=1}^{n} a_{i j}(x) \xi_{i} \xi_{j} \leq \Lambda|\xi|^{2} \omega(x), \quad \text { a.e. } x \in \Omega,
$$

for all $\xi \in \mathbb{R}^{n}$, and $\omega$ is an $A_{2}$-weight $(\lambda>0, \Lambda>0)$. Let $\mu \in \mathbb{R}$ and $f / \omega \in L^{2}(\Omega, \omega)$ and let $h$ be a real valued continuous function defined on $\mathbb{R} \times \mathbb{R}^{n}$. In this paper, we study the existence of weak solution of the BVP:

$$
\begin{gathered}
L u-\mu u g_{1}+h(u, \nabla u) g_{2}=f \quad \text { in } \Omega, \\
u=0 \quad \text { on } \partial \Omega,
\end{gathered}
$$

under suitable hypotheses on the functions $g_{1}, g_{2}$, and $h$. The present work is inspired by a semilinear problem in bounded domain given in the book by Zeidler [1]. In general, the Sobolev spaces $W^{k, p}(\Omega)$ without weights occur as spaces of solutions for elliptic and parabolic PDEs. For degenerate problems with various types of singularities in the coefficients it is natural to look for solutions in weighted Sobolev spaces; for example, see [2-8]. Section 2 deals with preliminaries and some basic results. Section 3 contains the main result and is about the existence of a weak solution to (3) in a suitable weighted Sobolev space.

\section{Preliminaries}

We need the following preliminaries for the ensuing study. Let $\Omega \subset \mathbb{R}^{n}$ be a bounded domain (open connected set). Let $\omega: \mathbb{R}^{n} \rightarrow \mathbb{R}^{+}$be a locally integrable function with $0<\omega<$ $\infty$ a.e. We say that $\omega$ belongs to the Muckenhoupt class $A_{p}$, $1<p<\infty$, or that $\omega$ is an $A_{p}$-weight, if there is a constant $c=c_{p, \omega}$ such that

$$
\left(\frac{1}{|B|} \int_{B} \omega(x) d x\right)\left(\frac{1}{|B|} \int_{B} \omega^{1 /(1-p)}(x) d x\right)^{p-1} \leq c,
$$

for all balls $B$ in $\mathbb{R}^{n}$, where $|\cdot|$ denotes the $n$-dimensional Lebesgue measure in $\mathbb{R}^{n}$. We assume that $\omega \in A_{p}, 1<p<\infty$. We will denote by $L^{p}(\Omega, \omega)(1 \leq p<\infty)$ the usual Banach space of measurable real valued functions, $f$, defined in $\Omega$ for which

$$
\|f\|_{p, \Omega}=\left(\int_{\Omega}|f(x)|^{p} \omega(x) d x\right)^{1 / p}<\infty .
$$


For $p \geq 1$ and a positive integer $k$, the weighted Sobolev space $W^{k, p}(\Omega, \omega)$ is defined by

$$
\begin{aligned}
& W^{k, p}(\Omega, \omega) \\
& \quad:=\left\{u \in L^{p}(\Omega, \omega): D^{\alpha} u \in L^{p}(\Omega, \omega), 1 \leq|\alpha| \leq k\right\}
\end{aligned}
$$

with the associated norm

$$
\|u\|_{k, p, \Omega}=\|u\|_{p, \Omega}+\sum_{1 \leq|\alpha| \leq k}\left\|D^{\alpha} u\right\|_{p, \Omega} .
$$

In order to avoid too many suffices, at each step, a generic constant is denoted by $c$ or $C_{\Omega}$. We need the following result.

Proposition 1 (the weighted Sobolev inequality). Let $\Omega \subset \mathbb{R}^{n}$ be a bounded domain and let $\omega \in A_{p}(1<p<\infty)$. Then, there exist positive constants $C_{\Omega}$ and $\delta$ such that, for all $u \in C_{0}^{\infty}(\Omega)$ and all $k$ satisfying $1 \leq k \leq n /(n-1)+\delta$,

$$
\|u\|_{k p, \Omega} \leq C_{\Omega}\|\nabla u\|_{p, \Omega} .
$$

A proof of the above statement can be found in [5, Theorem 1.3].

For $p=2$ and $k=1$ in the above inequality, we have

$$
\|u\|_{2, \Omega} \leq C_{\Omega}\|u\|_{0,1,2},
$$

where

$$
\|u\|_{0,1,2}=\|\nabla u\|_{2, \Omega}:=\left(\int_{\Omega}|\nabla u(x)|^{2} \omega d x\right)^{1 / 2} .
$$

Further, we use function space $W_{0}^{1,2}(\Omega, \omega)$ which is defined as the closure of $C_{0}^{\infty}(\Omega)$ with respect to the norm $\|u\|_{0,1,2}$ (correctness of definition of this norm follows from inequality (9)). We also note that $W^{1,2}(\Omega, \omega)$ and $W_{0}^{1,2}(\Omega, \omega)$ are Hilbert spaces.

For more details on $A_{p}$-weight and weighted Sobolev spaces, we refer to $[5,7,9-11]$.

Proposition 2. Let $\omega \in A_{p}, 1<p<\infty$, and let $\Omega$ be a bounded open set in $\mathbb{R}^{n}$. If $u_{n} \rightarrow u$ in $L^{p}(\Omega, \omega)$, then there exist a subsequence $\left\{u_{n_{k}}\right\}$ and a function $v \in L^{p}(\Omega, \omega)$ such that

(i) $u_{n_{k}} \rightarrow u(x)$ as $n_{k} \rightarrow \infty$, w-a.e. on $\Omega$;

(ii) $\left|u_{n_{k}}(x)\right| \leq v(x)$, w-a.e. on $\Omega$.

Proof. The proof of this theorem follows in the lines of Theorem 2.8.1 in [12].

Let $(f \mid x)$ denote the value of linear functional $f$ at $x$.

Definition 3. Let $C, D: X \rightarrow X^{*}$ be the operators on the real separable reflexive Banach space $X$. Then,

(i) $C$ satisfies condition $(S)$ if

$$
\begin{array}{r}
u_{n} \rightarrow u, \quad \lim _{n \rightarrow \infty}\left(C u_{n}-C u \mid u_{n}-u\right)=0, \\
\text { implies } u_{n} \longrightarrow u,
\end{array}
$$

(ii) $C$ is demicontinuous if and only if $u_{n} \rightarrow u$ as $n \rightarrow \infty$ implies $C u_{n} \rightarrow C u$ as $n \rightarrow \infty$,

(iii) $C+D$ is asymptotically linear if $C$ is linear and

$$
\frac{\|D u\|}{\|u\|} \longrightarrow 0, \quad \text { as }\|u\| \longrightarrow \infty \text {. }
$$

In Section 3, we use the following result.

Proposition 4. Let $B, N: X \rightarrow X^{*}$ be operators on the real separable reflexive Banach space $X$. Then,

(1) the operator $B: X \rightarrow X^{*}$ is linear and continuous;

(2) the operator $N: X \rightarrow X^{*}$ is demicontinuous and bounded;

(3) $B+N$ is asymptotically linear;

(4) for each $T \in X^{*}$ and for each $t \in[0,1]$, the operator $A_{t}$ defined by $A_{t}(u):=B u+t(N u-T)$ satisfies condition (S) in $X$.

If $B u=0$ implies $u=0$, then, for each $T \in X^{*}$, the equation $B u+N u=T$ has a solution in $X$.

For a detailed proof of the above theorem, we refer to [13] or to [1, Theorem 29.C].

Definition 5. One says that $u \in W_{0}^{1,2}(\Omega, \omega)$ is a weak solution of (3) if

$$
\begin{aligned}
& \int_{\Omega} a_{i j} D_{i} u(x) D_{j} \phi(x) d x-\int_{\Omega} \mu u(x) g_{1}(x) \phi(x) d x \\
& \quad+\int_{\Omega} h(u(x), \nabla u(x)) g_{2}(x) \phi(x) d x=\int_{\Omega} f(x) \phi(x) d x .
\end{aligned}
$$

for every $\phi \in W_{0}^{1,2}(\Omega, \omega)$.

We need the following hypotheses for further study.

$\left(H_{1}\right)$ Let $(\eta, \xi) \mapsto h(\eta, \xi)$ be continuous in $\mathbb{R} \times \mathbb{R}^{n}$, where $h$ is a bounded function (i.e., for a constant $A>0$, let $|h(t)| \leq$ $A, \forall t \in \mathbb{R})$.

$\left(H_{2}\right)$ Let $\omega \in A_{2}$. Assume $g_{1} / \omega \in L^{\infty}(\Omega), g_{2} / \omega \in$ $L^{2}(\Omega, \omega)$, and $f / \omega \in L^{2}(\Omega, \omega)$.

$\left(H_{3}\right)$ Assume $g_{2}\left(h(\eta, \xi)-h\left(\eta^{\prime}, \xi^{\prime}\right)\right)\left(\eta-\eta^{\prime}\right) \geq 0$, where $\eta, \eta^{\prime} \in \mathbb{R}$ and $\xi, \xi^{\prime} \in \mathbb{R}^{n}$.

We define the functionals $B_{1}, B_{2}$ : $W_{0}^{1,2}(\Omega, \omega) \times$ $W_{0}^{1,2}(\Omega, \omega) \rightarrow \mathbb{R}$ by

$$
\begin{aligned}
B_{1}(u, \phi)= & \int_{\Omega} a_{i j} D_{i} u(x) D_{j} \phi(x) d x \\
& -\int_{\Omega} \mu u(x) g_{1}(x) \phi(x) d x, \\
B_{2}(u, \phi)= & \int_{\Omega} h(u(x), \nabla u(x)) g_{2}(x) \phi(x) d x .
\end{aligned}
$$

Also define $T: W_{0}^{1,2}(\Omega, \omega) \rightarrow \mathbb{R}$ by

$$
T(\phi)=\int_{\Omega} f(x) \phi(x) d x .
$$


A function $u \in W_{0}^{1,2}(\Omega, \omega)$ is a weak solution of (3) if

$$
B_{1}(u, \phi)+B_{2}(u, \phi)=T(\phi), \quad \forall \phi \in W_{0}^{1,2}(\Omega, \omega) .
$$

By noting $\left|a_{i j}(x)\right| \leq c \omega(x)$ and by Hölder's inequality, we get

$$
\begin{aligned}
\left|B_{1}(u, \phi)\right| \leq & \int_{\Omega}\left|a_{i j}(x)\right|\left|D_{i} u(x)\right|\left|D_{j} \phi(x)\right| d x \\
& +|\mu| \int_{\Omega}|u(x)||\phi(x)|\left|g_{1}(x)\right| d x \\
\leq & c \int_{\Omega} \sum_{i, j=1}^{n}\left|D_{i} u(x)\right|\left|D_{j} \phi(x)\right| \omega(x) d x \\
& +|\mu| \int_{\Omega}|u(x)||\phi(x)|\left|\frac{g_{1}(x)}{\omega(x)}\right| \omega(x) d x \\
\leq & c\left(\int_{\Omega} \sum_{i=1}^{n}\left|D_{i} u(x)\right|^{2} \omega(x) d x\right)^{1 / 2} \\
& \times\left(\int_{\Omega} \sum_{j=1}^{n}\left|D_{j} \phi(x)\right|^{2} \omega(x) d x\right)^{1 / 2} \\
& +|\mu|\left\|\frac{g_{1}}{\omega}\right\| \|_{\infty}\left(\int_{\Omega}|u(x)|^{2} \omega(x) d x\right)^{1 / 2} \\
& \times\left(\int_{\Omega}|\phi(x)|^{2} \omega(x) d x\right)^{1 / 2} \\
\leq & \left.+C_{\Omega}|\mu| \mid \frac{g_{1}}{\omega} \|_{\infty}\right)\|u\|_{0,1,2}\|\phi\|_{0,1,2}
\end{aligned}
$$

where $C_{\Omega}$ is a constant arising out of $(9)$. Now, $B_{1}(\cdot, \cdot)$ is linear and bounded. Then, there exists an operator

$$
B: W_{0}^{1,2}(\Omega, \omega) \longrightarrow\left[W_{0}^{1,2}(\Omega, \omega)\right]^{*}
$$

defined by $(B u \phi)=B_{1}(u, \phi)$, for all $u, \phi \in W_{0}^{1,2}(\Omega, \omega)$. Also, by $\left(H_{1}\right)$ and $\left(H_{2}\right)$, it follows from Hölder's inequality that

$$
\begin{aligned}
\left|B_{2}(u, \phi)\right| & =\left|\int_{\Omega} g_{2}(x) h(u(x), \nabla u(x)) \phi(x) d x\right| \\
& \leq A \int_{\Omega}\left|\frac{g_{2}(x)}{\omega(x)}\right||\phi(x)| \omega(x) d x \\
& \leq A\left\|\frac{g_{2}}{\omega}\right\|_{2, \Omega}\|\phi\|_{2, \Omega} \\
& \leq A\left\|\frac{g_{2}}{\omega}\right\|_{2, \Omega}\|\phi\|_{0,1,2},
\end{aligned}
$$

and hence by the weighted Sobolev inequality (9)

$$
\left|B_{2}(u, \phi)\right| \leq C_{\Omega}|u|_{0,1,2}|\phi|_{0,1,2}, \quad \forall u, \phi \in W_{0}^{1,2}(\Omega, \omega) .
$$

Now $B_{2}(u, \cdot)$ is linear and so there exists an operator $N$ : $W_{0}^{1,2}(\Omega, \omega) \rightarrow\left[W_{0}^{1,2}(\Omega, \omega)\right]^{*}$ such that

$$
(N u \mid \phi)=B_{2}(u, \phi), \quad \forall u, \phi \in W_{0}^{1,2}(\Omega, \omega) .
$$

Further, we have

$$
\begin{aligned}
|T(\phi)| & \leq \int_{\Omega}|f(x)||\phi(x)| d x \\
& \leq\left\|\frac{f}{\omega}\right\|_{2, \Omega}\|\phi\|_{2, \Omega} \leq C_{\Omega}\left\|\frac{f}{\omega}\right\|_{2, \Omega}|\phi|_{0,1,2} .
\end{aligned}
$$

Then, problem (3) is equivalent to solving the operator equation

$$
B u+N u=T, \quad \forall u \in W_{0}^{1,2}(\Omega, \omega)
$$

\section{Main Results}

The main result of this section is to establish the existence of a solution for the degenerate nonlinear elliptic BVP (3), when $\mu>0$ is not an eigenvalue of

$$
\begin{gathered}
L u-\mu u(x) g_{1}(x)=0 \quad \text { in } \Omega, \\
u=0 \quad \text { on } \partial \Omega,
\end{gathered}
$$

with certain restrictions. Also, two results are established related to the cases when $g_{1}$ does not change sign.

Theorem 6. Assume the hypotheses $\left(H_{1}\right)-\left(H_{3}\right)$ and the inequality

$$
\lambda>\mu C_{\Omega}\left\|\frac{g_{1}}{\omega}\right\|_{\infty},
$$

where $C_{\Omega}$ is a constant arising out of (9). Let $\mu>0$ not be an eigenvalue of (24). Then, the BVP (3) has a solution $u \in$ $W_{0}^{1,2}(\Omega, \omega)$.

Proof. Idea of proof is such. First we write a weak solution of the BVP (3) as solution of operator equation

$$
u \in W_{0}^{1,2}(\Omega, \omega): B u+N u=T \quad \text { in }\left[W_{0}^{1,2}(\Omega, \omega)\right]^{*},
$$

where $T \in\left[W_{0}^{1,2}(\Omega, \omega)\right]^{*}, B: W_{0}^{1,2}(\Omega, \omega) \rightarrow\left[W_{0}^{1,2}(\Omega, \omega)\right]^{*}$ is linear and continuous, and $N: W_{0}^{1,2}(\Omega, \omega) \rightarrow$ $\left[W_{0}^{1,2}(\Omega, \omega)\right]^{*}$ is demicontinuous and bounded and satisfies few more conditions. Further, we put Proposition 4 to this operator equation. The realization of this idea is split into 5 steps for convenience.

Step 1. We note that the operator $B$ is linear. It follows from (17) and (20) that the operators $B, N$ are bounded.

Step 2. Let $u_{k} \rightarrow u$ in $W_{0}^{1,2}(\Omega, \omega)$. We now claim

$$
N u_{k} \rightarrow N u \quad \text { in } W_{0}^{1,2}(\Omega, \omega) \text { as } k \longrightarrow \infty
$$

or

$$
\begin{aligned}
& \left|\left(N u_{k}-N u \mid \phi\right)\right| \longrightarrow 0, \\
& \forall \phi \in W_{0}^{1,2}(\Omega, \omega) \text { as } k \longrightarrow \infty .
\end{aligned}
$$


If $u_{k} \rightarrow u$ in $W_{0}^{1,2}(\Omega, \omega)$, then $\left|\nabla u_{k}\right| \rightarrow|\nabla u|$ in $L^{2}(\Omega, \omega)$ and $u_{k} \rightarrow u$ in $L^{2}(\Omega, \omega)$. Using Proposition 2, there exist a subsequence $\left\{u_{n_{k}}\right\}$ and functions $v_{1}$ and $v_{2}$ in $L^{2}(\Omega, \omega)$ such that

$$
\begin{aligned}
& u_{k}(x) \longrightarrow u(x) \quad \omega \text {-a.e. in } \Omega, \\
& \left|u_{n_{k}}(x)\right| \leq v_{1}(x) ; \quad \omega \text {-a.e. in } \Omega, \\
& \nabla u_{k}(x) \longrightarrow \nabla u(x) \quad \omega \text {-a.e. in } \Omega, \\
& \left|\nabla u_{n_{k}}(x)\right| \leq v_{2}(x) ; \quad \omega \text {-a.e. in } \Omega .
\end{aligned}
$$

Now

$$
\begin{aligned}
\left|\left(N u_{k}-N u \mid \phi\right)\right|= & \mid \int_{\Omega} h\left(u_{k}(x), \nabla u_{k}(x)\right) \\
& -h(u(x), \nabla u(x)) g_{2}(x) \phi_{k}(x) \mid .
\end{aligned}
$$

Since $h$ is bounded, we get

$$
\begin{aligned}
& \left|h\left(u_{k}(x), \nabla u_{k}(x)\right)-h(u(x), \nabla u(x)) g_{2}(x) \phi(x)\right| \\
& \quad \leq 2 A\left|g_{2} \phi\right| .
\end{aligned}
$$

Also,

$$
\int_{\Omega}\left|g_{2} \phi\right| \leq\left\|\frac{g_{2}}{\omega}\right\|_{2, \Omega}\|\phi\|_{2, \Omega}<\infty
$$

By $\left(H_{1}\right)$ and (29), we infer that

$$
\begin{array}{r}
{\left[h\left(u_{k}(x), \nabla u_{k}(x)\right)-h(u(x), \nabla u(x))\right] g_{2}(x) \phi(x) \longrightarrow 0} \\
\text { a.e in } \Omega, \text { as } k \longrightarrow \infty .
\end{array}
$$

Letting $k \rightarrow \infty$, by dominated convergence theorem, we obtain

$$
\begin{aligned}
& \left|\left(N u_{k}-N u \mid \phi\right)\right| \\
& =\mid \int_{\Omega}\left[h\left(u_{k}(x), \nabla u_{k}(x)\right)-h(u(x), \nabla u(x))\right] \\
& \quad \times g_{2}(x) \phi(x) \mid \longrightarrow 0 .
\end{aligned}
$$

Hence, we have claim (28) or equivalently $N$ is demicontinuous.

Step 3. Next, we claim that $B+N$ is asymptotically linear. Since $h$ is bounded, we observe that, for all $u \in W_{0}^{1,2}(\Omega, \omega)$,

$$
|(N u \mid \phi)|=\left|B_{2}(u, \phi)\right| \leq A C_{\Omega}\left\|\frac{g_{2}}{\omega}\right\|_{2, \Omega}\|\phi\|_{0,1,2}
$$

which implies $\|N u\| \leq C^{\prime}$, where $C^{\prime}=A C_{\Omega}\left\|g_{2} / \omega\right\|_{2, \Omega}$. Consequently,

$$
\frac{\|N u\|}{\|u\|_{0,1,2}} \longrightarrow 0 \quad \text { as }\|u\|_{0,1,2} \longrightarrow \infty
$$

which shows that $B+N$ is asymptotically linear.

Step 4. We denote $A_{t}(u)=B u+t(N u-T), t \in[0,1]$. Let $u_{k} \rightarrow u$ in $W_{0}^{1,2}(\Omega, \omega)$ and

$$
\begin{aligned}
0 & =\lim _{k \rightarrow \infty}\left(B u_{k}+t\left(N u_{k}-T\right)-\left(B u+t(N u-T) \mid u_{k}-u\right)\right) \\
& =\lim _{k \rightarrow \infty}\left(B u_{k}-B u+t\left(N u_{k}-N u\right) \mid u_{k}-u\right) \\
& =\lim _{k \rightarrow \infty}\left(B u_{k}-B u \mid u_{k}-u\right)+t \lim _{k \rightarrow \infty}\left(N u_{k}-N u \mid u_{k}-u\right) .
\end{aligned}
$$

We claim that $u_{k} \rightarrow u$ strongly in $W_{0}^{1,2}(\Omega, \omega)$ or $A_{t}(u)$ satisfies condition (S). By (2) and inequality (9) of Proposition 1, it follows that

$$
\begin{aligned}
B_{1} & \left(u_{k}-u, u_{k}-u\right) \\
& =\int_{\Omega} a_{i j} D_{i}\left(u_{k}-u\right) D_{j}\left(u_{k}-u\right) d x-\int_{\Omega} \mu\left(u_{k}-u\right)^{2} g_{1} d x \\
& \geq \lambda \int_{\Omega}\left|\nabla\left(u_{k}-u\right)\right|^{2} \omega d x-\int_{\Omega} \mu\left(u_{k}-u\right)^{2} g_{1} d x \\
& \geq\left(\lambda-\mu C_{\Omega}\left\|\frac{g_{1}}{\omega}\right\|_{\infty}\right) \int_{\Omega}\left|\nabla\left(u_{k}-u\right)\right|^{2} \omega d x \\
& =\beta_{\Omega}\left\|u_{k}-u\right\|_{0,1,2}^{2},
\end{aligned}
$$

where $\beta_{\Omega}=\left(\lambda-\mu C_{\Omega}\left\|g_{1} / \omega\right\|_{\infty}\right)$, a positive constant depending on $\Omega$. Since $B$ is linear, from (38) we have

$$
\begin{aligned}
\left(B u_{k}-B u \mid u_{k}-u\right)= & \left(B\left(u_{k}-u\right) \mid u_{k}-u\right) \\
& =B_{1}\left(u_{k}-u, u_{k}-u\right) \\
& \geq \beta_{\Omega}\left\|u_{k}-u\right\|_{0,1,2}^{2} .
\end{aligned}
$$

By hypothesis $\left(H_{3}\right)$, we have now

$$
\begin{aligned}
& \left(N u_{n}-N u \mid u_{n}-u\right) \\
& \quad=\int_{\Omega}\left[g_{2} h\left(u_{k}, \nabla u_{k}\right)-h(u, \nabla u)\right]\left(u_{n}-u\right) \geq 0 .
\end{aligned}
$$

From (37), (39), and (40), we note

$$
0 \leq \beta_{\Omega_{k}} \lim _{k \rightarrow \infty}\left\|u_{k}-u\right\|_{0,1,2}^{2}=0 .
$$

Since, by condition (25), $\beta_{\Omega}>0$, we have

$$
\left\|u_{k}-u\right\|_{0,1,2}^{2} \longrightarrow 0, \quad \text { as } k \longrightarrow \infty \text {. }
$$


Consequently, $\left\|u_{k}-u\right\|_{0,1,2} \rightarrow 0$, as $k \rightarrow \infty$, which implies that, for each $t \in[0,1], A_{t}(u)$ satisfies condition (S).

Step 5. Since, by given hypothesis, $\mu>0$ is not an eigenvalue of (24), $B u=0$ implies $u=0$. By Proposition $4, B u+N u=T$ has a solution $u \in W_{0}^{1,2}(\Omega, \omega)$ which equivalently shows that the BVP (3) has a solution $u \in W_{0}^{1,2}(\Omega, \omega)$.

Remark 7. In the following results, we dispense with condition (25), when $g_{1}$ does not change sign. The two results are related to the cases when $g_{1}>0$ with $\mu<0$ and $g_{1}<0$ with $\mu>0$.

The proof of the following results is similar to Theorem 6 and, hence, we give a sketch of the proof.

Theorem 8. Suppose that $\left(H_{1}\right)-\left(H_{3}\right)$ hold. Let $g_{1}>0$ and $\mu<$ 0 ; then the BVP (3) has a solution $u \in W_{0}^{1,2}(\Omega, \omega)$.

Proof. As in Theorem 6, the basic idea is to reduce the problem (3) to an operator equation $\mathrm{Bu}+\mathrm{Nu}=\mathrm{T}$. We note that the operator $B$ is linear and continuous and $N$ is bounded. Let $u_{k} \rightarrow u$ in $W_{0}^{1,2}(\Omega, \omega)$ and as in (37)

$$
\begin{aligned}
0= & \lim _{k \rightarrow \infty}\left(A_{t}\left(u_{k}\right)-A_{t}(u) \mid u_{k}-u\right) \\
= & \lim _{k \rightarrow \infty}\left(B u_{k}-B u \mid u_{k}-u\right) \\
& +t \lim _{k \rightarrow \infty}\left(\left(N u_{k}-N u\right) \mid u_{k}-u\right) .
\end{aligned}
$$

Since $\mu<0$ and $g_{1}>0$, then, by (2) and the weighted Sobolev inequality (9), it follows that

$$
\begin{aligned}
& B_{1}\left(u_{k}-u, u_{k}-u\right) \\
& =\int_{\Omega} a_{i j} D_{i}\left(u_{k}-u\right) D_{j}\left(u_{k}-u\right) d x \\
& \quad-\int_{\Omega} \mu\left(u_{k}-u\right)^{2} g_{1} d x \\
& \geq \lambda \int_{\Omega}\left|\nabla\left(u_{k}-u\right)\right|^{2} \omega d x-\int_{\Omega} \mu\left(u_{k}-u\right)^{2} g_{1} d x \\
& \geq \lambda \int_{\Omega}\left|\nabla\left(u_{k}-u\right)\right|^{2} \omega d x=\lambda\left\|u_{k}-u\right\|_{0,1,2}^{2} .
\end{aligned}
$$

Since $B$ is linear, from (44) we have

$$
\begin{aligned}
& \left(B u_{k}-B u \mid u_{k}-u\right) \\
& \quad=\left(B\left(u_{k}-u\right) \mid u_{k}-u\right) \\
& \quad=B_{1}\left(u_{k}-u, u_{k}-u\right) \geq \lambda\left\|u_{k}-u\right\|_{0,1,2}^{2} .
\end{aligned}
$$

By hypothesis $\left(\mathrm{H}_{3}\right)$, we have now

$$
\begin{aligned}
\left(N u_{n}-N u \mid u_{n}-u\right) & \\
=\int_{\Omega}[ & g_{2}(x) h\left(u_{k}(x), \nabla u_{k}(x)\right) \\
& \quad-h(u(x), \nabla u(x))]\left(u_{n}-u\right) \geq 0 .
\end{aligned}
$$

From (43), (45), and (46), we note that

$$
0 \leq \lambda \lim _{k \rightarrow \infty}\left\|u_{k}-u\right\|_{0,1,2}^{2}=0
$$

Since $\lambda>0$, we have $\left\|u_{k}-u\right\|_{0,1,2}^{2} \rightarrow 0$ and hence $\| u_{k}-$ $u \|_{0,1,2} \rightarrow 0$, as $k \rightarrow \infty$, which implies that, for each $t \in$ $[0,1], A_{t}(u)$ satisfies condition (S). Also, we note that $B+N$ is asymptotically linear. Now $B u=0$ implies

$$
\int_{\Omega} a_{i j}\left|D_{i} u(x)\right|^{2} d x-\int_{\Omega} \mu u^{2}(x) g_{1}(x) d x=0
$$

or

$$
\begin{aligned}
\lambda \int_{\Omega}|D u(x)|^{2} d x & \leq \int_{\Omega} a_{i j}\left|D_{i} u(x)\right|^{2} d x \\
& =\mu \int_{\Omega} u^{2}(x) g_{1}(x) d x
\end{aligned}
$$

which shows that $u=0$ (since $\mu<0$ and $g_{1}>0$ ). By Proposition 4, Bu $+N u=T$ has a solution $u \in$ $W_{0}^{1,2}(\Omega, \omega)$.

With suitable changes in the proof of Theorem 8 , we arrive at the following result.

Theorem 9. Let the hypotheses of Theorem 8 hold, except that $g_{1}<0$ and $\mu>0$. Then, the BVP (3) has a solution $u \in$ $W_{0}^{1,2}(\Omega, \omega)$.

Proof. The proof of this result is in the same lines to that of Theorem 8 and hence omitted.

Remark 10. In Theorems 8 and 9, we have studied the BVP (3) both positive and negative values of $\mu$. In Theorem 9, with positive value of $\mu$ we do not need the extra condition (25) at the cost of $g_{1}$ negative.

\section{Conflict of Interests}

The author declares that there is no conflict of interests regarding the publication of this paper.

\section{References}

[1] E. Zeidler, Nonlinear Functional Analysis and Its ApplicationsPart II/B, Springer, New York, NY, USA, 1990.

[2] A. C. Cavalheiro, "Existence of solutions in weighted Sobolev spaces for some degenerate semilinear elliptic equations," Applied Mathematics Letters, vol. 17, no. 4, pp. 387-391, 2004.

[3] B. Franchi and R. Serapioni, "Pointwise estimates for a class of strongly degenerate elliptic operators: a geometrical approach," Annali della Scuola Normale Superiore di Pisa, vol. 14, no. 4, pp. 527-568, 1987.

[4] E. Fabes, C. Kenig, and R. Serapioni, "The local regularity of solutions of degenerate elliptic equations," Communications in Partial Differential Equations, vol. 7, no. 1, pp. 77-116, 1982.

[5] E. Fabes, D. Jerison, and C. Kenig, "The Wiener test for degenerate elliptic equations," Annales de l'Institut Fourier, vol. 32, no. 3, pp. 151-182, 1982. 
[6] S. Chanillo and R. L. Wheeden, "Weighted Poincaré and Sobolev inequalities and estimates for weighted Peano maximal functions," American Journal of Mathematics, vol. 107, no. 5, pp. 1191-1226, 1985.

[7] V. C. Piat and F. S. Cassano, "Relaxation of degenerate variational integrals," Nonlinear Analysis: Theory, Methods \& Applications, vol. 22, no. 4, pp. 409-424, 1994.

[8] V. Raghavendra and R. Kar, "Existence of weak solutions for degenerate semilinear elliptic equations in unbounded domains," Electronic Journal of Differential Equations, vol. 160, pp. 1-13, 2009.

[9] B. O. Turesson, Nonlinear Potential Theory and Weighted Sobolev Spaces, vol. 1736 of Lecture Notes in Mathematics, Springer, Berlin, Germany, 2000.

[10] J. García-Cuerva and J. L. R. de Francia, Weighted Norm Inequalities and Related Topics, vol. 116 of North-Holland Mathematics Studies, North-Holland, Amsterdam, The Netherlands, 1985.

[11] J. Heinonen, T. Kilpeläinen, and O. Martio, Nonlinear Potential Theory of Degenerate Elliptic Equations, Oxford Mathematical Monographs, Clarendon Press, Oxford, UK, 1993.

[12] A. Kufner, O. John, and S. Fučik, Functions Spaces, Noordhoff, Leyden, The Netherlands, 1977.

[13] P. Hess, "On the Fredholm alternative for nonlinear functional equations in Banach spaces," Proceedings of the American Mathematical Society, vol. 33, pp. 55-61, 1972. 


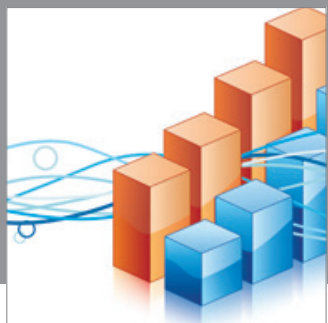

Advances in

Operations Research

mansans

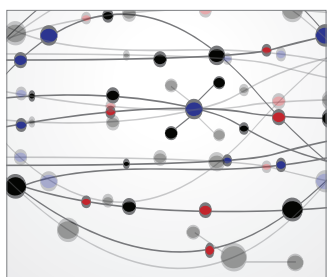

The Scientific World Journal
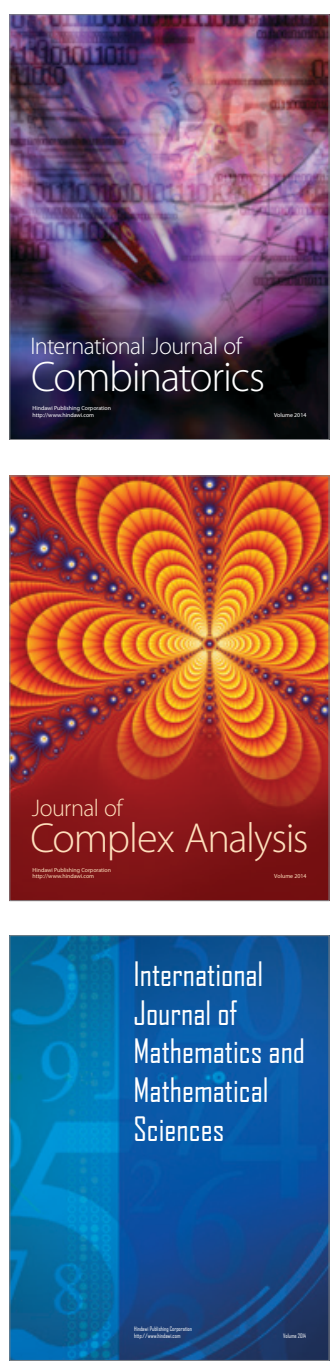
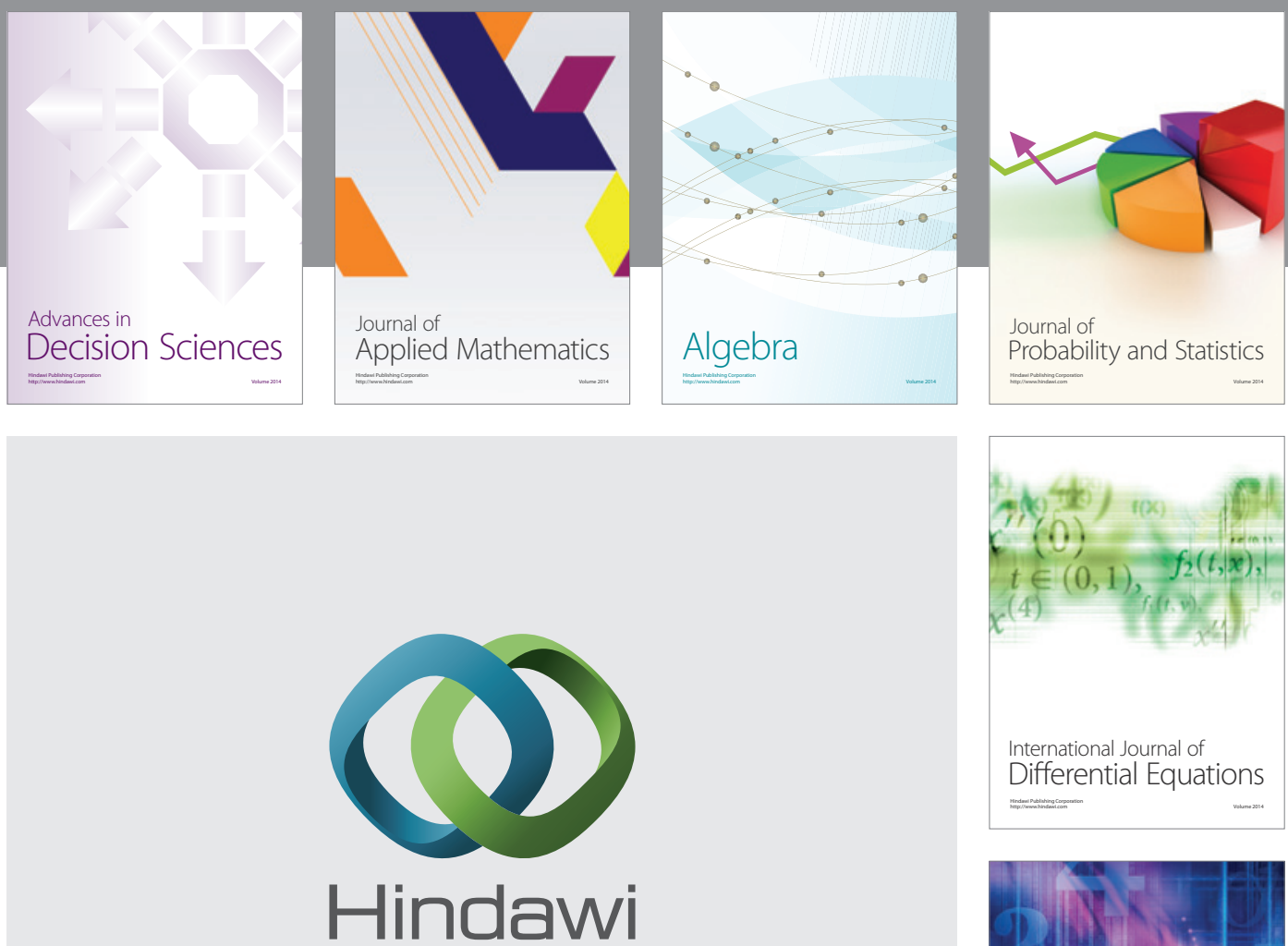

Submit your manuscripts at http://www.hindawi.com
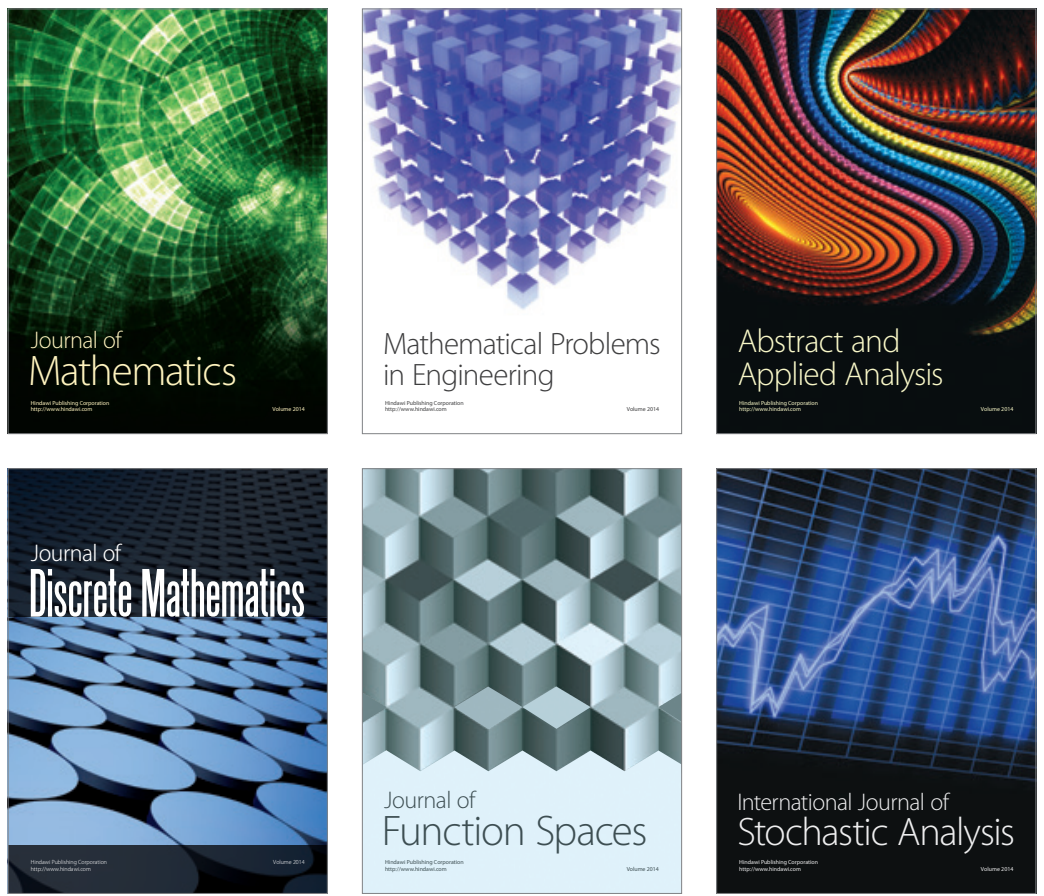

Journal of

Function Spaces

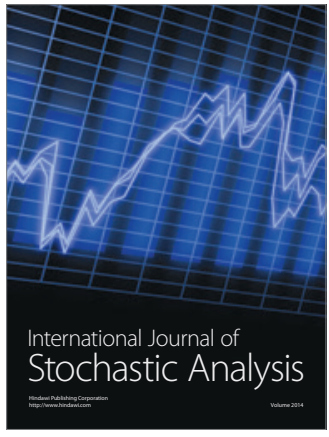

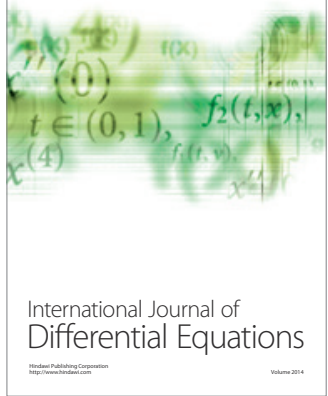
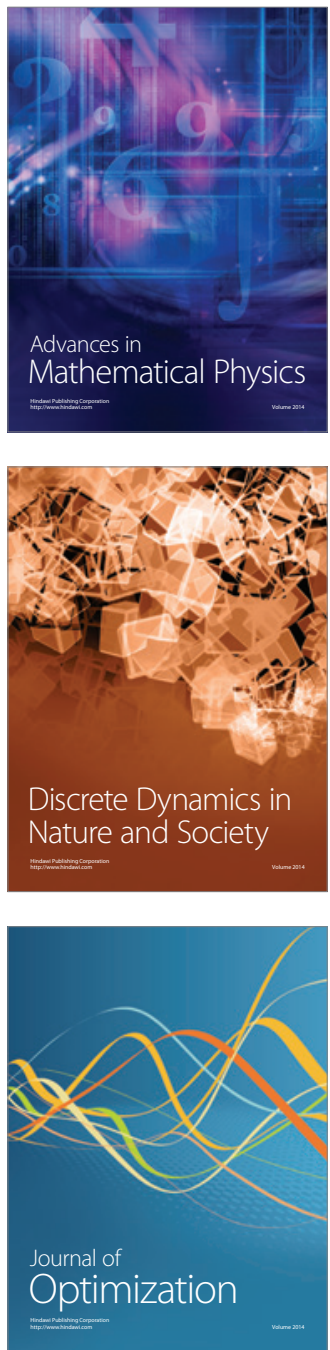